\title{
ON TAUBERIAN REMAINDER THEOREMS FOR CESÀRO SUMMABILITY METHOD OF NONINTEGER ORDER
}

\author{
Ü. TOTUR AND M. A. OKUR
}

Received 18 June, 2014

\begin{abstract}
In this paper, we prove some Tauberian remainder theorems for Cesàro summability method of noninteger order $\alpha>-1$.
\end{abstract}

2010 Mathematics Subject Classification: 40E05; 40G05

Keywords: Tauberian remainder theorem, $\lambda$-bounded series, $(C, \alpha)$ summability, Cesàro means

\section{INTRODUCTION}

Let $A_{n}^{\alpha}$ be defined by the generating function $(1-x)^{-\alpha-1}=\sum_{n=0}^{\infty} A_{n}^{\alpha} x^{n}$, $(|x|<1)$, where $\alpha>-1$. For a real sequence $u=\left(u_{n}\right)$, the Cesàro means of the sequence $\left(u_{n}\right)$ of noninteger order $\alpha$ are defined by

$$
\sigma_{n}^{(\alpha)}(u)=\frac{1}{A_{n}^{\alpha}} \sum_{j=0}^{n} A_{n-j}^{\alpha-1} u_{j} .
$$

We say that a sequence $\left(u_{n}\right)$ is $(C, \alpha)$ summable to a finite number $s$, where $\alpha>-1$ if

$$
\lim _{n \rightarrow \infty} \sigma_{n}^{(\alpha)}(u)=s
$$

and we write $u_{n} \rightarrow s(C, \alpha)$. We denote the backward difference of $\left(u_{n}\right)$, by $\Delta u_{n}=$ $u_{n}-u_{n-1}$, with $\Delta u_{0}=u_{0}$. We define $\tau_{n}(u)=n \Delta u_{n} \quad(n=0,1,2, .$.$) and indicate$ $\tau_{n}^{(\alpha)}(u)$ as $(C, \alpha)$ mean of $\left(\tau_{n}(u)\right)$.

Note that if taking $\alpha=k$ where $k$ is a nonnegative integer, then we obtain the $(C, k)$ summability method and for $\alpha=0$, the $(C, 0)$ summability is ordinary convergence.

The $(C, \alpha)$ summability method is regular, more generally, if a sequence $\left(u_{n}\right)$ is $(C, \alpha)$ summable to $s$, where $\alpha>-1$ and $\beta \geq \alpha$ for $\alpha, \beta$, then $\left(u_{n}\right)$ is also $(C, \beta)$ summable to $s$. However, the converse is not always true. The converse of this statement is valid under some conditions called Tauberian conditions. Any theorem which states that convergence of a sequence follows from a summability method and some Tauberian condition(s) is said to be a Tauberian theorem. Recently, a number 
of authors such as Estrada and Vindas [4,5], Natarajan [15], Çanak et al. [2], Erdem and Çanak [3], Çanak and Erdem [1] have investigated Tauberian theorems for several summability methods.

For a sequence $\left(u_{n}\right)$ and for each integer $m \geq 1$,

$$
(n \Delta)_{m} u_{n}=n \Delta\left((n \Delta)_{m-1} u_{n}\right)
$$

where $(n \Delta)_{0} u_{n}=u_{n}$ and $(n \Delta)_{1} u_{n}=n \Delta u_{n}$.

For $\alpha>-1$, the identity

$$
\tau_{n}^{(\alpha)}(u)=n \Delta \sigma_{n}^{(\alpha)}(u)
$$

was proved by Kogbetliantz [9]. Note that $\tau_{n}^{(0)}(u)=\tau_{n}(u)$.

The identity

$$
\sigma_{n}^{(\alpha)}(u)-\sigma_{n}^{(\alpha+1)}(u)=\frac{1}{\alpha+1} \tau_{n}^{(\alpha+1)}(u)
$$

is used in the various steps of proofs (see [10]).

Çanak et al. [2] represent the identity

$$
n \Delta \tau_{n}^{\alpha+1}=(\alpha+1)\left(\tau_{n}^{\alpha}-\tau_{n}^{\alpha+1}\right),
$$

for $\alpha>-1$.

Erdem and Çanak [3] prove that for $\alpha>-1$ and any integer $k \geq 1$

$$
(n \Delta)_{k} \tau_{n}^{(\alpha+k)}(u)=\sum_{j=1}^{k}(-1)^{j+1} A_{k}^{(j)}(\alpha) n \Delta \tau_{n}^{(\alpha+j)}(u),
$$

where $A_{k}^{(j)}(\alpha)=a_{k}^{(j-1)}(\alpha)+a_{k}^{(j)}(\alpha), \quad a_{k}^{(0)}(\alpha)=0$ and

$$
a_{k}^{(j)}(\alpha)=\Pi_{i=j+1}^{k}(\alpha+i) \sum_{\substack{j \leq t_{1}, t_{2}, \ldots, t_{j-1} \leq k \\ r<s \Rightarrow t_{r} \leq t_{s}}}\left(\alpha+t_{1}\right)\left(\alpha+t_{2}\right) \ldots\left(\alpha+t_{j-1}\right) .
$$

\section{TAUBERIAN REMAINDER THEOREMS}

Let $\lambda=\left(\lambda_{n}\right)$ be a nondecreasing sequence of positive numbers such that $\lambda_{n} \rightarrow \infty$. A sequence $\left(u_{n}\right)$ is called bounded with the rapidity $\left(\lambda_{n}\right)$ (in short $\lambda$-bounded) if

$$
\lambda_{n}\left(u_{n}-s\right)=O(1),
$$

with $\lim _{n \rightarrow \infty} u_{n}=s$. Let

$$
m^{\lambda}=\left\{u=\left(u_{n}\right) \mid \lim _{n \rightarrow \infty} u_{n}=s \quad \text { and } \quad \lambda_{n}\left(u_{n}-s\right)=O(1)\right\} .
$$

A sequence $\left(u_{n}\right)$ is called $\lambda$-bounded by the $(C, \alpha)$ method of summability if

$$
\lambda_{n}\left(\sigma_{n}^{(\alpha)}(u)-s\right)=O(1)
$$

with $\lim _{n \rightarrow \infty} \sigma_{n}^{(\alpha)}(u)=s$. Shortly, we write $u \in\left((C, \alpha), m^{\lambda}\right)$. 
G. Kangro [7] introduced the concepts of Tauberian remainder theorems using summability with given rapidity $\lambda$. G. Kangro [8] and Tammeraid [16, 17] proved some Tauberian remainder theorems for several summability method, such as Riesz, Cesàro, Hölder and Euler-Knopp methods. Recently, various authors have represented some Tauberian remainder theorems (see [12,13]). In [18], Tammeraid proved some Tauberian remainder theorems in which the $(C, \alpha)$ summability method is used. Tauberian remainder theorems have also been studied by many authors via the Fourier integral method. [6,11]

Meronen and Tammeraid [14] proved the following Tauberian remainder theorems:

Theorem 1. Let the condition

$$
\lambda_{n} \tau_{n}^{(1)}(u)=O(1)
$$

be satisfied. If $u \in\left((C, 1), m^{\lambda}\right)$, then $u \in m^{\lambda}$.

Theorem 2. Let the conditions

$$
\begin{array}{r}
\lambda_{n} \tau_{n}(u)=O(1), \\
\lambda_{n} n \Delta \tau_{n}^{(1)}(u)=O(1)
\end{array}
$$

be satisfied. If $u \in\left((C, 1), m^{\lambda}\right)$, then $u \in m^{\lambda}$.

The main purpose of this paper is to prove several Tauberian remainder theorems for Cesàro summability method of noninteger order $\alpha>-1$. Our main theorems improve Theorem 1 and Theorem 2 given by Meronen and Tammeraid [14].

\section{A Lemma}

We require the following lemma to be used in the proofs of main theorems.

Lemma 1. Let $\alpha>-1$. For any integer $k \geq 2$,

$$
\begin{aligned}
& (n \Delta)_{k} \tau_{n}^{(\alpha+k)}(u)=B_{1,1-\alpha} \tau_{n}^{(\alpha)}(u)-B_{1,1} \sigma_{n}^{(\alpha)}(u)+B_{1,1} \sigma_{n}^{(\alpha+1)}(u) \\
& \quad+\sum_{j=2}^{k}\left(B_{j, j-1} \sigma_{n}^{(\alpha+j-2)}(u)-2 B_{j, j-\frac{1}{2}} \sigma_{n}^{(\alpha+j-1)}(u)+B_{j, j} \sigma_{n}^{(\alpha+j)}(u)\right),
\end{aligned}
$$

where $B_{m, l}=(\alpha+m)(\alpha+l)(-1)^{m+1} A_{k}^{(m)}(\alpha)$ and $A_{k}^{(j)}(\alpha)=a_{k}^{(j-1)}(\alpha)+a_{k}^{(j)}(\alpha)$, $a_{k}^{(0)}(\alpha)=0$ and

$$
a_{k}^{(j)}(\alpha)=\Pi_{i=j+1}^{k}(\alpha+i) \sum_{\substack{j+1 \leq t_{1}, t_{2}, \ldots, t_{j-1} \leq k \\ r<s \Rightarrow t_{r} \leq t_{s}}}\left(\alpha+t_{1}\right)\left(\alpha+t_{2}\right) \ldots\left(\alpha+t_{j-1}\right) .
$$


Proof. From identity (1.6), we have

$$
\begin{aligned}
(n \Delta)_{k} \tau_{n}^{(\alpha+k)}(u) & =\sum_{j=1}^{k}(-1)^{j+1} A_{k}^{(j)}(\alpha) n \Delta \tau_{n}^{(\alpha+j)}(u) \\
& =A_{k}^{(1)}(\alpha) n \Delta \tau_{n}^{(\alpha+1)}(u)+\sum_{j=2}^{k}(-1)^{j+1} A_{k}^{(j)}(\alpha) n \Delta \tau_{n}^{(\alpha+j)}(u)
\end{aligned}
$$

It follows from identity (1.5) that

$$
\begin{aligned}
(n \Delta)_{k} \tau_{n}^{(\alpha+k)}(u)= & (\alpha+1) A_{k}^{(1)}(\alpha)\left(\tau_{n}^{(\alpha)}(u)-\tau_{n}^{(\alpha+1)}(u)\right) \\
& +\sum_{j=2}^{k}(\alpha+j)(-1)^{j+1} A_{k}^{(j)}(\alpha)\left(\tau_{n}^{(\alpha+j-1)}(u)-\tau_{n}^{(\alpha+j)}(u)\right) .
\end{aligned}
$$

By identity (1.4), we can write the above equation as

$$
\begin{gathered}
(n \Delta)_{k} \tau_{n}^{(\alpha+k)}(u)=(\alpha+1) A_{k}^{(1)}(\alpha) \tau_{n}^{(\alpha)}(u)-(\alpha+1)^{2} A_{k}^{(1)}(\alpha)\left(\sigma_{n}^{(\alpha)}(u)-\sigma_{n}^{(\alpha+1)}(u)\right) \\
+\sum_{j=2}^{k}(\alpha+j)(-1)^{j+1} A_{k}^{(j)}(\alpha)\left((\alpha+j-1)\left(\sigma_{n}^{(\alpha+j-2)}(u)-\sigma_{n}^{(\alpha+j-1)}(u)\right)\right. \\
\left.-(\alpha+j)\left(\sigma_{n}^{(\alpha+j-1)}(u)-\sigma_{n}^{(\alpha+j)}(u)\right)\right) .
\end{gathered}
$$

Therefore,

$$
\begin{aligned}
& (n \Delta)_{k} \tau_{n}^{(\alpha+k)}(u) \\
& =(\alpha+1) A_{k}^{(1)}(\alpha) \tau_{n}^{(\alpha)}(u)-(\alpha+1)^{2} A_{k}^{(1)}(\alpha) \sigma_{n}^{(\alpha)}(u)+(\alpha+1)^{2} A_{k}^{(1)}(\alpha) \sigma_{n}^{(\alpha+1)}(u) \\
& \quad+\sum_{j=2}^{k}\left((\alpha+j)(\alpha+j-1)(-1)^{j+1} A_{k}^{(j)}(\alpha) \sigma_{n}^{(\alpha+j-2)}(u)\right. \\
& \quad-(\alpha+j)(\alpha+j-1)(-1)^{j+1} A_{k}^{(j)}(\alpha) \sigma_{n}^{(\alpha+j-1)}(u) \\
& \left.\quad-(\alpha+j)^{2}(-1)^{j+1} A_{k}^{(j)}(\alpha) \sigma_{n}^{(\alpha+j-1)}(u)+(\alpha+j)^{2}(-1)^{j+1} A_{k}^{(j)}(\alpha) \sigma_{n}^{(\alpha+j)}(u)\right) .
\end{aligned}
$$

Hence, we have

$$
\begin{aligned}
& (n \Delta)_{k} \tau_{n}^{(\alpha+k)}(u) \\
& =(\alpha+1) A_{k}^{(1)}(\alpha) \tau_{n}^{(\alpha)}(u)-(\alpha+1)^{2} A_{k}^{(1)}(\alpha) \sigma_{n}^{(\alpha)}(u)+(\alpha+1)^{2} A_{k}^{(1)}(\alpha) \sigma_{n}^{(\alpha+1)}(u) \\
& \quad+\sum_{j=2}^{k}\left((\alpha+j)(\alpha+j-1)(-1)^{j+1} A_{k}^{(j)}(\alpha) \sigma_{n}^{(\alpha+j-2)}(u)\right. \\
& \quad-(\alpha+j)(2 \alpha+2 j-1)(-1)^{j+1} A_{k}^{(j)}(\alpha) \sigma_{n}^{(\alpha+j-1)}(u)
\end{aligned}
$$




$$
\left.+(\alpha+j)^{2}(-1)^{j+1} A_{k}^{(j)}(\alpha) \sigma_{n}^{(\alpha+j)}(u)\right) .
$$

Taking $(\alpha+m)(\alpha+l)(-1)^{m+1} A_{k}^{(m)}(\alpha)=B_{m, l}$, we obtain

$$
\begin{aligned}
& (n \Delta)_{k} \tau_{n}^{(\alpha+k)}(u)=B_{1,1-\alpha} \tau_{n}^{(\alpha)}(u)-B_{1,1} \sigma_{n}^{(\alpha)}(u)+B_{1,1} \sigma_{n}^{(\alpha+1)}(u) \\
& \quad+\sum_{j=2}^{k}\left(B_{j, j-1} \sigma_{n}^{(\alpha+j-2)}(u)-2 B_{j, j-\frac{1}{2}} \sigma_{n}^{(\alpha+j-1)}(u)+B_{j, j} \sigma_{n}^{(\alpha+j)}(u)\right) .
\end{aligned}
$$

Thus, we conclude that Lemma 1 is true for each integer $k \geq 2$.

\section{MAIN RESUltS}

In the main theorems, we prove some Tauberian remainder theorems to recover $\lambda$ bounded by the $(C, \alpha)$ summability of a sequence out of $\lambda$-bounded by the $(C, \alpha+j)$ summability for $j=1,2$ and any integer $j=k$, and some suitable conditions. In special cases of main theorems, we obtain some classical type Tauberian remainder theorems for the $(C, 1)$ summability method.

Theorem 3. Let the conditions

$$
\lambda_{n} n \Delta \tau_{n}^{(\alpha+1)}(u)=O(1),
$$

and

$$
\lambda_{n} \tau_{n}^{(\alpha)}(u)=O(1)
$$

be satisfied for $\alpha>-1$. If $u \in\left((C, \alpha+1), m^{\lambda}\right)$, then $u \in\left((C, \alpha), m^{\lambda}\right)$.

Proof. From identity (1.5), we have

$$
\begin{aligned}
\lambda_{n} n \Delta \tau_{n}^{(\alpha+1)}(u) & =\lambda_{n}(\alpha+1)\left(\tau_{n}^{(\alpha)}(u)-\tau_{n}^{(\alpha+1)}(u)\right) \\
& =\lambda_{n}(\alpha+1) \tau_{n}^{(\alpha)}(u)-\lambda_{n}(\alpha+1) \tau_{n}^{(\alpha+1)}(u) .
\end{aligned}
$$

From identity (1.4), we obtain

$$
\lambda_{n} n \Delta \tau_{n}^{(\alpha+1)}(u)=\lambda_{n}(\alpha+1) \tau_{n}^{(\alpha)}(u)-\lambda_{n}(\alpha+1)^{2}\left(\sigma_{n}^{(\alpha)}(u)-\sigma_{n}^{(\alpha+1)}(u)\right) .
$$

Rewritten the above equation, we have

$$
\begin{aligned}
\lambda_{n}(\alpha+1)^{2}\left(\sigma_{n}^{(\alpha)}(u)-s\right)= & \lambda_{n}(\alpha+1)^{2}\left(\sigma_{n}^{(\alpha+1)}(u)-s\right) \\
& +\lambda_{n}(\alpha+1) \tau_{n}^{(\alpha)}(u)-\lambda_{n} n \Delta \tau_{n}^{(\alpha+1)}(u) .
\end{aligned}
$$

Using (4.1) and (4.2), we get

$$
\lambda_{n}(\alpha+1)^{2}\left(\sigma_{n}^{(\alpha)}(u)-s\right)=O(1)+O(1)+O(1)=O(1) .
$$

Therefore, $\lambda_{n}\left(\sigma_{n}^{(\alpha)}(u)-s\right)=O(1)$. That means $u \in\left((C, \alpha), m^{\lambda}\right)$.

Notice that taking $\alpha=0$, we obtain Theorem 2 . 
Proposition 1. Let the conditions

$$
\begin{aligned}
\lambda_{n}(n \Delta)_{2} \tau_{n}^{(\alpha+2)}(u) & =O(1), \\
\lambda_{n} \tau_{n}^{(\alpha)}(u) & =O(1),
\end{aligned}
$$

and

$$
\lambda_{n}\left(\sigma_{n}^{(\alpha+2)}(u)-s\right)=O(1)
$$

be satisfied for $\alpha>-1$. If $u \in\left((C, \alpha+1), m^{\lambda}\right)$, then $u \in\left((C, \alpha), m^{\lambda}\right)$.

Proof. Taking $k=2$ in Lemma 1, we have

$$
\begin{aligned}
\lambda_{n}(n \Delta)_{2} \tau_{n}^{(\alpha+2)}(u)= & \lambda_{n}(\alpha+2)(\alpha+1)\left(\tau_{n}^{(\alpha)}(u)-\tau_{n}^{(\alpha+1)}(u)\right) \\
& -\lambda_{n}(\alpha+2)^{2}\left(\tau_{n}^{(\alpha+1)}(u)-\tau_{n}^{(\alpha+2)}(u)\right) \\
= & \lambda_{n}(\alpha+2)(\alpha+1) \tau_{n}^{(\alpha)}(u)-\lambda_{n}(\alpha+2)(\alpha+1) \tau_{n}^{(\alpha+1)}(u) \\
& -\lambda_{n}(\alpha+2)^{2} \tau_{n}^{(\alpha+1)}(u)+\lambda_{n}(\alpha+2)^{2} \tau_{n}^{(\alpha+2)}(u) .
\end{aligned}
$$

From identity (1.4), we get

$$
\begin{aligned}
\lambda_{n}(n \Delta)_{2} \tau_{n}^{(\alpha+2)}(u) \\
=\lambda_{n}(\alpha+2)(\alpha+1) \tau_{n}^{(\alpha)}(u)-\lambda_{n}(\alpha+2)(\alpha+1)\left((\alpha+1)\left(\sigma_{n}^{(\alpha)}(u)-\sigma_{n}^{(\alpha+1)}(u)\right)\right) \\
\quad-\lambda_{n}(\alpha+2)^{2}\left((\alpha+1)\left(\sigma_{n}^{(\alpha)}(u)-\sigma_{n}^{(\alpha+1)}(u)\right)\right) \\
\quad+\lambda_{n}(\alpha+2)^{2}\left((\alpha+2)\left(\sigma_{n}^{(\alpha+1)}(u)-\sigma_{n}^{(\alpha+2)}(u)\right)\right) \\
=\lambda_{n}(\alpha+2)(\alpha+1) \tau_{n}^{(\alpha)}(u)-\lambda_{n}(\alpha+1)^{2}(\alpha+2) \sigma_{n}^{(\alpha)}(u) \\
\quad+\lambda_{n}(\alpha+1)^{2}(\alpha+2) \sigma_{n}^{(\alpha+1)}(u)-\lambda_{n}(\alpha+2)^{2}(\alpha+1) \sigma_{n}^{(\alpha)}(u) \\
\quad+\lambda_{n}(\alpha+2)^{2}(\alpha+1) \sigma_{n}^{(\alpha+1)}(u)+\lambda_{n}(\alpha+2)^{3} \sigma_{n}^{(\alpha+1)}(u)-\lambda_{n}(\alpha+2)^{3} \sigma_{n}^{(\alpha+2)}(u) \\
=\lambda_{n}(\alpha+2)(\alpha+1) \tau_{n}^{(\alpha)}(u)-\lambda_{n}(\alpha+1)(\alpha+2)(2 \alpha+3) \alpha_{n}^{(\alpha)}(u) \\
\quad+\lambda_{n}\left((\alpha+2)^{3}+(\alpha+2)^{2}(\alpha+1)+(\alpha+1)^{2}(\alpha+2)\right) \sigma_{n}^{(\alpha+1)}(u) \\
\quad-\lambda_{n}(\alpha+2)^{3} \sigma_{n}^{(\alpha+2)}(u) .
\end{aligned}
$$

Rewritten the above equation, we have

$$
\begin{aligned}
& \lambda_{n}(\alpha+1)(\alpha+2)(2 \alpha+3)\left(\sigma_{n}^{(\alpha)}(u)-s\right) \\
& =-\lambda_{n} n \Delta \tau_{n}^{(\alpha+2)}(u)+\lambda_{n}(\alpha+2)(\alpha+1) \tau_{n}^{(\alpha)}(u)+\lambda_{n}\left((\alpha+2)^{3}+(\alpha+2)^{2}(\alpha+1)\right. \\
& \left.\quad+(\alpha+1)^{2}(\alpha+2)-s\right) \sigma_{n}^{(\alpha+1)}(u)-\lambda_{n}\left((\alpha+2)^{3}-s\right) \sigma_{n}^{(\alpha+2)}(u) .
\end{aligned}
$$

Using (4.3), (4.4) and (4.5), we get

$$
\lambda_{n}(\alpha+1)(\alpha+2)(2 \alpha+3)\left(\sigma_{n}^{(\alpha)}(u)-s\right)=O(1)+O(1)+O(1)=O(1) .
$$


Therefore, $\lambda_{n}\left(\sigma_{n}^{(\alpha)}(u)-s\right)=O(1)$. That means $u \in\left((C, \alpha), m^{\lambda}\right)$.

Now, we represent a Tauberian remainder theorem which generalizes Theorem 3 and Proposition 1.

Theorem 4. Let the conditions

$$
\begin{aligned}
\lambda_{n}(n \Delta)_{k} \tau_{n}^{(\alpha+k)}(u) & =O(1), \\
\lambda_{n} \tau_{n}^{(\alpha)}(u) & =O(1),
\end{aligned}
$$

and

$$
\lambda_{n}\left(\sigma_{n}^{(\alpha+j)}(u)-s\right)=O(1) \quad \text { for } \quad 2 \leq j \leq k
$$

be satisfied for $\alpha>-1$. If $u \in\left((C, \alpha+1), m^{\lambda}\right)$, then $u \in\left((C, \alpha), m^{\lambda}\right)$.

Proof. From Lemma 1 we have

$$
\begin{aligned}
\lambda_{n}(n \Delta)_{k} \tau_{n}^{(\alpha+k)}(u)=B_{1,1-\alpha} \lambda_{n} \tau_{n}^{(\alpha)}(u)-B_{1,1} \lambda_{n} \sigma_{n}^{(\alpha)}(u)+B_{1,1} \lambda_{n} \sigma_{n}^{(\alpha+1)}(u) \\
\quad+\lambda_{n} \sum_{j=2}^{k}\left(B_{j, j-1} \sigma_{n}^{(\alpha+j-2)}(u)-2 B_{j, j-\frac{1}{2}} \sigma_{n}^{(\alpha+j-1)}(u)+B_{j, j} \sigma_{n}^{(\alpha+j)}(u)\right),
\end{aligned}
$$

Rewritten the above equation, we have

$$
\begin{aligned}
& B_{1,1} \lambda_{n}\left(\sigma_{n}^{(\alpha)}(u)-s\right) \\
& =B_{1,1-\alpha} \lambda_{n} \tau_{n}^{(\alpha)}(u)-\lambda_{n}(n \Delta)_{k} \tau_{n}^{(\alpha+k)}(u)+B_{1,1} \lambda_{n}\left(\sigma_{n}^{(\alpha+1)}(u)-s\right) \\
& \quad+\lambda_{n} \sum_{j=2}^{k} B_{j, j-1}\left(\sigma_{n}^{(\alpha+j-2)}(u)-s\right)-\lambda_{n} \sum_{j=2}^{k} 2 B_{j, j-\frac{1}{2}}\left(\sigma_{n}^{(\alpha+j-1)}(u)-s\right) \\
& \quad+\lambda_{n} \sum_{j=2}^{k} B_{j, j}\left(\sigma_{n}^{(\alpha+j)}(u)-s\right) .
\end{aligned}
$$

Using (4.6), (4.7) and (4.8), we get

$$
B_{1,1} \lambda_{n}\left(\sigma_{n}^{(\alpha)}(u)-s\right)=O(1)+O(1)+O(1)+O(1)+O(1)+O(1)=O(1) .
$$

Therefore, $\lambda_{n}\left(\sigma_{n}^{(\alpha)}(u)-s\right)=O(1)$. That means $u \in\left((C, \alpha), m^{\lambda}\right)$.

Theorem 5. Let the condition

$$
\lambda_{n} \tau_{n}^{(\alpha+j+1)}(u)=O(1) \quad \text { for } \quad 0 \leq j \leq k-1,
$$

be satisfied for $\alpha>-1$. If $u \in\left((C, \alpha+k), m^{\lambda}\right)$, then $u \in\left((C, \alpha), m^{\lambda}\right)$.

Proof. Suppose that $u \in\left((C, \alpha+k), m^{\lambda}\right)$. Taking $j=k-1$ in (4.9), it follows from the idendity

$$
\tau_{n}^{(\alpha+k)}(u)=(\alpha+k)\left(\sigma_{n}^{(\alpha+k-1)}(u)-\sigma_{n}^{(\alpha+k)}(u)\right)
$$


that we obtain

$$
\begin{aligned}
\lambda_{n}(\alpha+k)\left(\sigma_{n}^{(\alpha+k-1)}(u)-s\right) & =\lambda_{n} \tau_{n}^{(\alpha+k)}(u)+\lambda_{n}(\alpha+k)\left(\sigma_{n}^{\alpha+k}(u)-s\right) \\
& =O(1)+O(1)=O(1)
\end{aligned}
$$

then we obtain $\lambda_{n}\left(\sigma_{n}^{(\alpha+k-1)}-s\right)=O(1)$. Hence, that means

$$
u \in\left((C, \alpha+k-1), m^{\lambda}\right) \text {. }
$$

From identity (1.4), we have

$$
\tau_{n}^{(\alpha+k-1)}(u)=(\alpha+k-1)\left(\sigma_{n}^{(\alpha+k-2)}(u)-\sigma_{n}^{(\alpha+k-1)}(u)\right) .
$$

Taking $j=k-2$ in (4.9), we obtain

$$
\begin{aligned}
& \lambda_{n}(\alpha+k-1)\left(\alpha_{n}^{(\alpha+k-2)}(u)-s\right) \\
& =\lambda_{n} \tau_{n}^{(\alpha+k-1)}(u)+\lambda_{n}(\alpha+k-1)\left(\sigma_{n}^{(\alpha+k-1)}(u)-s\right)=O(1)+O(1)=O(1)
\end{aligned}
$$

Therefore we have

$$
u \in\left((C, \alpha+k-2), m^{\lambda}\right)
$$

Continuing in this way, we obtain that

$$
u \in\left((C, \alpha+1), m^{\lambda}\right) \text {. }
$$

Taking $j=0$ in (4.9), we obtain $\lambda_{n} \tau_{n}^{(\alpha+1)}=O(1)$. From identity (1.4), we have

$$
\begin{aligned}
\lambda_{n}(\alpha+1)\left(\sigma_{n}^{(\alpha)}(u)-s\right) & =\lambda_{n} \tau_{n}^{(\alpha+1)}(u)+\lambda_{n}(\alpha+1)\left(\sigma_{n}^{(\alpha+1)}(u)-s\right) \\
& =O(1)+O(1)=O(1)
\end{aligned}
$$

This completes the proof.

Theorem 6. Let the condition

$$
\lambda_{n}(n \Delta)_{j} \tau_{n}^{(\alpha+j)}(u)=O(1) \quad \text { for } \quad 0 \leq j \leq k,
$$

be satisfied for $\alpha>-1$. If $u \in\left((C, \alpha+k), m^{\lambda}\right)$, then $u \in\left((C, \alpha), m^{\lambda}\right)$.

Proof. By identity (1.6) for $k=1$, it follows

$$
\begin{aligned}
\lambda_{n} n \Delta \tau_{n}^{(\alpha+1)}(u) & =\lambda_{n}(\alpha+1)\left(\tau_{n}^{(\alpha)}(u)-\tau_{n}^{(\alpha+1)}(u)\right) \\
& =\lambda_{n}(\alpha+1) \tau_{n}^{(\alpha)}(u)-\lambda_{n}(\alpha+1) \tau_{n}^{(\alpha+1)}(u) .
\end{aligned}
$$

Taking $j=0$ and $j=1$ in (4.10), we obtain

$$
\lambda_{n} \tau_{n}^{(\alpha+1)}(u)=O(1)
$$

From identity (1.6) for $k=2$, we get

$$
\begin{aligned}
\lambda_{n}(n \Delta)_{2} \tau_{n}^{(\alpha+2)}(u)= & \lambda_{n}(\alpha+2)(\alpha+1)\left(\tau_{n}^{(\alpha)}(u)-\tau_{n}^{(\alpha+1)}(u)\right) \\
& -\lambda_{n}(\alpha+2)^{2}\left(\tau_{n}^{(\alpha+1)}(u)-\tau_{n}^{(\alpha+2)}(u)\right) .
\end{aligned}
$$


Taking $j=0$ and $j=2$ in (4.10), we obtain

$$
\lambda_{n} \tau_{n}^{(\alpha+2)}(u)=O(1)
$$

Continuing in this way, by Lemma 1 , we obtain

$$
\begin{aligned}
\lambda_{n}(n \Delta)_{k} \tau_{n}^{(\alpha+k)}(u)= & (\alpha+1) A_{k}^{(1)}(\alpha) \lambda_{n}\left(\tau_{n}^{(\alpha)}(u)-\tau_{n}^{(\alpha+1)}(u)\right) \\
& +\lambda_{n} \sum_{j=2}^{k}(\alpha+j)(-1)^{j+1} A_{k}^{(j)}(\alpha)\left(\tau_{n}^{(\alpha+j-1)}(u)-\tau_{n}^{(\alpha+j)}(u)\right) .
\end{aligned}
$$

Taking $j=0$ and $j=k$ in (4.10), we obtain

$$
\lambda_{n} \tau_{n}^{(\alpha+k)}(u)=O(1)
$$

The conditions in Theorem 5 hold, the proof is completed.

\section{ACKNOWLEDGEMENT}

The authors are grateful to the anonymous referee who has made invaluable suggestions that helped to improve this paper.

\section{REFERENCES}

[1] Çanak, İ. and Erdem, Y., "On Tauberian theorems for $(A)(C, \alpha)$ summability method," App. Math. and Comp., vol. 218, no. 6, pp. 2829-2836, 2011, doi: 10.1016/j.amc.2011.08.026.

[2] Çanak, İ. and Erdem, Y. and Totur, Ü., "Some Tauberian theorems for $(A)(C, \alpha)$ summability method," Math. Comput. Modell., vol. 52, no. 5-6, pp. 738-743, 2010, doi: 10.1016/j.mcm.2010.05.001.

[3] Erdem, Y. and Çanak, I., "A Tauberian theorem for $(A)(C, \alpha)$ summability," Comput. Math. Appl., vol. 60, no. 11, pp. 2920-2925, 2010, doi: 10.1016/j.camwa.2010.09.047.

[4] Estrada, R. and Vindas J., "On Tauber's second Tauberian theorem," Tohoku Math. J., vol. 64, no. 4, pp. 539-560, 2012.

[5] Estrada, R. and Vindas J., "Distributional versions of Littlewood's Tauberian theorem," Czechoslovak Math. J., vol. 63, no. 2, pp. 403-420, 2013, doi: 10.1007/s10587-013-0025-1.

[6] Ganelius, T. H., "Tauberian remainder theorems," Lecture Notes in Mathematics, vol. 232, 1971.

[7] Kangro, G., "Summability factors of Bohr-Hardy type for a given rate. I, II," Eesti NSV Tead. Akad. Toimetised Fü̈̈s.-Mat., vol. 18, pp. 137-146, 387-395, 1969.

[8] Kangro, G., "Summability factors for series that are $\lambda$-bounded by the Riesz and Cesàro methods," Tartu Riikl. Ül. Toimetised Vih., vol. 277, pp. 136-154, 1971.

[9] Kogbetliantz, E., "Sur le séries absolument sommables par la méthode des moyennes arihtmétiques," Bulletin sc. Math., vol. 49, pp. 234-251, 1925.

[10] Kogbetliantz, E., "Sommation des séries et intégrals divergents par les moyennes arithmétiques et typiques," Memorial Sci. Math., vol. 51, pp. 1-84, 1931.

[11] J. Koreavar, Tauberian theory. Berlin: Springer, 2004, vol. 329.

[12] Meronen, O. and Tammeraid, I., "Generalized linear methods and gap Tauberian remainder theorems," Math. Model. Anal., vol. 13, no. 2, pp. 223-232, 2008, doi: 10.3846/13926292.2008.13.223-232.

[13] Meronen, O. and Tammeraid, I., "Several theorems on $\lambda$-summable series," Math. Model. Anal., vol. 15, no. 1, pp. 97-102, 2010, doi: 10.3846/1392-6292.2010.15.97-102. 
[14] Meronen, O. and Tammeraid, I., "General control modulo and Tauberian remainder theorems for (C,1) summability," Math. Model. Anal., vol. 18, no. 1, pp. 97-102, 2013, doi: $10.3846 / 13926292.2013 .758674$.

[15] Natarajan, P. N., “A Tauberian theorem for weighted means," Bull. Pure Appl. Math., vol. 6, no. 1, pp. 45-48, 2012.

[16] Tammeraid, I., "Tauberian theorems with a remainder term for the Cesàro and Hölder summability methods," Tartu Riikl. Ül. Toimetised No., vol. 277, pp. 161-170, 1971.

[17] Tammeraid, I., "Tauberian theorems with a remainder term for the Euler-Knopp summability method," Tartu Riikl. Ül. Toimetised No., vol. 277, pp. 171-182, 1971.

[18] Tammeraid, I., "Two Tauberian remainder theorems for the Cesàro method of summability," Proc. Estonian Acad. Sci. Phys. Math., vol. 49, no. 4, pp. 225-232, 2000.

Authors' addresses

Ü. Totur

Adnan Menderes University, Department of Mathematics, 09010, Aydin, Turkey

E-mail address: utotur@adu.edu.tr

M. A. Okur

Adnan Menderes University, Department of Mathematics, 09010, Aydin, Turkey

E-mail address: mali.okureadu.edu.tr 